Salavera Bordás, C. \& Usán, P. (2018). Uso del humor e inteligencia emocional en estudiantes de Secundaria. Revista Electrónica Interuniversitaria de Formación del Profesorado, 21(3), 109-122.

\title{
Uso del humor e inteligencia emocional en estudiantes de Secundaria
}

Carlos Salavera Bordás y Pablo Usán Supervía

Universidad de Zaragoza

\section{Resumen}

En la presente investigación, se evaluó a 1304 estudiantes de Secundaria (14-18 años) con el objetivo de analizar la relación entre uso del humor e inteligencia emocional. Los resultados muestran como los chicos hacen un mayor uso del humor y de la gestión de las emociones en sus relaciones sociales. El uso del humor no mostró efecto en atención, mientras que el humor contraproducente presentó un efecto indirecto y significativo $(B=.22, p<.005)$ con comprensión y el humor de automejora mostró un efecto directo y significativo $(B=.53$, $p<.001 ; R^{2}=44.5 \%$ ) con regulación. Los datos mostraron como un $68.02 \%$ de los adolescentes mostraron bajos niveles de inteligencia emocional y del uso del humor. Como conclusión, afirmar que los datos de la investigación evidencian la necesidad de trabajar programas de entrenamiento en el uso del humor y la inteligencia emocional que les puedan proporcionar soporte emocional y servir en la gestión de conflictos.

\section{Palabras clave}

Uso del humor; inteligencia emocional; estudiantes; adolescentes.

\section{Contacto:}

Carlos Salavera, salavera@unizar.es, Facultad de Educación Universidad de Zaragoza c/ Pedro Cerbuna, 12. 50009 Zaragoza.. 


\title{
Use of humour and emotional intelligence in Secondary Education students.
}

\begin{abstract}
In the present investigation, 1304 Secondary students (14-18 years old) were evaluated in order to analyze the relationship between the use of humor and emotional intelligence. The results show how boys make greater use of humor and the management of emotions in their social relationships. The use of humor showed no effect in Attention, while the counterproductive mood presented an indirect and significant effect $(B=.22, p<.005)$ with Understanding and the mood of self-improvement showed a direct and significant effect ( $B$ $=.53, \mathrm{p}<.001 ; \mathrm{R} 2=44.5 \%)$ with Regulation. The data showed that $68.02 \%$ of adolescents showed low levels of emotional intelligence and the use of humor. As a conclusion, to affirm that the data of the investigation show the need to work training programs in the use of humor and emotional intelligence that can provide emotional support and serve in the management of conflicts.
\end{abstract}

\section{Key words}

Use of humor; emotional intelligence; students; adolescents.

\section{Introducción}

El uso del humor ha cobrado especial importancia en los últimos años debido a su relación con la salud física y psicológica de la persona (Capps, 2006; García-Larrauri, Manzano, Muñoz, Hernández y Cuetos, 2010; Heintz, 2017), encontrándose relaciones con la reducción del dolor, la satisfacción con la vida, mejora de la condición física y mejores niveles de salud en general (Maiolino \& Kuipers, 2014; Panish, 2002). El humor se encuentra implicado en algunas de las tareas psicológicas más complejas e importantes siendo responsable de distintos beneficios sociales, cognitivos comportamentales, tanto en tareas individuales como en aspectos interpersonales (Carretero-Dios, Benítez, Delgado-Rico, Ruch, \& LópezBenítez, 2014; Cassaretto \& Martínez, 2009; Gignac, Karatamoglou, Wee \& Palacios, 2014; Kozbetz \& Nishioka, 2010; Martin, 2003; Sirigatti, Penzo, Giannetti, Casale, \& Stefanile, 2016).

Por otra parte, la inteligencia emocional se encuentra asociada con el funcionamiento y desempeño social, siendo responsable de las relaciones interpersonales y de cuestiones como la satisfacción, la autoestima o el optimismo (Di Fabio \& Kenny, 2016; Extremera \& Rey, 2016; Freudenthaler \& Neubauer, 2005; Garay, Mendiguren, Álvarez, \& Vizcarra, 2017; Matthews, Zeidner \& Roberts, 2007; Salavera \& Usán, 2017; Stough, Saklofske \& Parker, 2009).

Algunos autores han sugerido que el humor juega un papel importante en el campo educativo y en las relaciones interpersonales, como un método de mejora de las interacciones positivas y facilitando, de este modo, las autorevelaciones y el acercamiento 
social, desactivando tensiones y conflictos e incrementando las buenas relaciones (Carbelo, 2011; Ford, Lappi, \& Holden, 2016; Lefcourt, 2001; Long \& Graesser, 1988). Así mismo, los estilos negativos de humor, como las bromas de mal gusto o el sarcasmo, muestran un impacto negativo en las relaciones sociales (Martin, Puhli-Doris, Larse, Gray \& Weir, 2003; Vrabel, Zeigler-Hill, \& Shango, 2017; Weinstein, Hodgins \& Ostvik-White, 2011).

Diversos estudios han encontrado correlaciones entre el uso del humor y variables de las relaciones sociales como satisfacción en las relaciones de pareja, soporte social percibido y calidad en las relaciones sociales (Airenti, 2016; Martin et al., 2003; Nezlek \& Derks, 2001; Ziv \& Gadish, 1989).

Esta visión del uso del humor como un elemento de competencia social sugiere que éste puede se puede encontrar, de alguna forma, relacionado con el constructo de inteligencia emocional (IE). Estudios recientes han señalado cómo existen líneas de conexión entre IE (concretamente en la gestión de esas emociones) y la calidad de las interacciones sociales (Caballero, Alcaraz, Alonso, \& Yuste, 2016; Lopes, Brackett, Nezlek, Schutz, Sellin \& Salovey, 2004; López-Pérez, Wilson, Dellaria, \& Gummerum, 2016). Muchos de los usos socialmente relevantes, tienen que ver con la conciencia emocional y la regulación de las emociones, componentes de esa IE. De manera concisa, el humor puede entenderse como un fenómeno relativamente complejo en el que concurren diferentes elementos procesos de índole cognitivo y afectivo, así como un importante mecanismo de regulación emocional, y podría esperarse que correlacionase con aspectos de la IE, en concreto con la gestión de emociones, como un método de afrontamiento al estrés y mantenimiento de una perspectiva alegre frente a la adversidad (Martin, Kuiper, Olinger \& Dance, 1993), pero también como un mecanismo de ajuste psicosocial a lo largo de su proceso educativo (Fox, Hunter, \& Jones, 2016a; Fox, Hunter, \& Jones, 2016b).

Aunque existen evidencias de la relación entre el humor y los mecanismos de afrontamiento de estrés así como de las relaciones entre el humor y los estados de ánimo positivos y negativos (Lefcourt, 2001; Martin et al., 2003), apenas existen investigaciones sobre el uso del humor y la gestión de emociones tal y como las define el concepto de IE.

De este modo, el objetivo de la investigación se ha centrado en el análisis de la relación de los diferentes estilos de humor (afiliativo y de automejora, agresivo y autoagresivo), con la atención, comprensión y regulación de las emociones.

El estudio presenta dos hipótesis de partida: 1) el uso del humor y la inteligencia emocional de los adolescentes, se encuentran relacionados; y 2) el uso del sentido del humor utilizado por el sujeto, predice la inteligencia emocional autopercibida por el mismo.

\section{Metodología}

\section{Participantes}

El muestreo realizado fue de tipo estratificado siendo la unidad primaria la titularidad de los centros y la etapa de secundaria el nivel educativo ( $3^{\circ}, 4^{\circ}$ ESO y $1^{\circ}, 2^{\circ}$ Bachillerato). La muestra estuvo formada por 1304 estudiantes: 637 chicos (48.85\%) y 667 chicas (51.15\%) de 
ocho institutos públicos de Educación Secundaria de Zaragoza. La edad de los participantes fue de 14 a 18 años, con un media de 16.13 años (d.s. = 3.204) y se encontraban cursando $3^{\circ} \mathrm{y}$ $4^{\circ}$ de ESO y $1^{\circ}$ y $2^{\circ}$ de Bachillerato. Todos ellos participaron en el estudio de manera voluntaria, firmaron el consentimiento informado y se observaron las consideraciones éticas de la Declaración de Helsinki. Realizado el cálculo del tamaño de la muestra (Ato y Vallejo, 2015), con un nivel de confianza del $95 \%$ y un error muestral del $5 \%$, se obtuvo que la muestra final encuestada resultó representativa de la provincia de Zaragoza. El estudio se diseñó como un estudio retrospectivo ex - post facto.

\section{Instrumentos}

\section{Cuestionario de estilos de humor (HSQ) (Martin, Publio-Doris, Larsen, Gray \& Weir, 2003).}

El cuestionario adquiere el formato de autoinforme compuesto por 32 ítems, en una escala Likert de 7 puntos, que se erigen desde "totalmente diferente de mí" (1) a "totalmente igual a mí" (7). Consta de cuatro escalas de ocho ítems cada una: (afiliación: uso benigno del humor para facilitar las relaciones interpersonales; automejora: empleo de una visión humorística pero realista de la vida; agresividad: utilizado para sobresalir a expensas de otros; y descalificación personal: uso de un humor cínico divirtiendo a los demás a expensas de la propia persona). La prueba puede ser aplicada a adultos y adolescentes desde los 14 años de edad. Erickson y Feldstein (2007) obtuvieron buenos resultados con adolescentes desde los 12 años. Posee una fiabilidad alta (alfa $=.810)$. En la presente investigación, la fiabilidad de las escalas fue: Afiliación (alfa = .809); automejora (alfa = .823); agresivo (alfa = .786); y autodestructivo (alfa $=.794)$.

\section{Cuestionario TMMS-24 (Extremera, Fernández-Berrocal y Ramos, 2004)}

El TMMS-24 está basado en Trait Meta-Mood Scale (TMMS) del grupo de investigación de Salovey y Mayer. La escala original es una escala rasgo de formato Likert de 5 puntos que evalúa el meta-conocimiento de los estados emocionales. En concreto, las destrezas con las que podemos ser conscientes de nuestras propias emociones así como de nuestra capacidad para regularlas. La TMMS-24 contiene tres dimensiones claves de la IE (con 8 ítems cada una de ellas): atención emocional, comprensión de sentimientos y regulación emocional. Posee una fiabilidad alta (alfa $=0.84)$. En la presente investigación, la fiabilidad de las escalas fue: atención (alfa = .872); comprensión (alfa = .868); regulación (alfa = .854); con un total de la escala (alfa $=.852$ ).

\section{Procedimiento}

Para la selección de la muestra se solicitó la colaboración de los centros mediante llamada telefónica y, una vez confirmada la participación, se elaboró la lista de los centros participantes. En el momento de la entrega de cada escala, se procedió a explicar a los participantes el objetivo de la investigación, incidiendo en la importancia que tenía la cumplimentación de todos los ítems.

Los participantes tenían treinta minutos para completar los cuestionarios que se acaban de describir y el consentimiento informado. En todos los casos se les recordaba que la información recogida era anónima y confidencial. Los datos recogidos en el presente estudio se obtuvieron entre los meses de febrero a abril de 2016. 
Para el análisis estadístico de los datos se ha empleado el programa estadístico SPSS 22.0. Comprobada la normalidad de la muestra e igualdad de varianzas se optó por técnicas paramétricas. Se realizó un análisis descriptivo para cada una de las variables. En todos los casos se trabajó y se han considerado significativas aquellas diferencias con un valor de $p<$ 0.05. Los contrastes se plantearon de forma bilateral. Para el contraste de hipótesis de dos grupos se utilizó la $t$ de Student y para más de dos grupos la ANOVA unifactorial. Se estableció un conglomerado de medias para obtener clúster que nos permitiesen clasificar a los participantes en distintos grupos en función de su uso del humor e inteligencia emocional. Finalmente, el establecimiento de ecuaciones predictoras de los factores de inteligencia emocional en función de los estilos de humor, se realizó mediante la técnica de regresión logística, siguiendo el procedimiento de regresión por pasos hacia delante basado en el estadístico de Wald.

\section{Resultados}

Se analizaron las diferencias en cuánto a género en estilos del humor e inteligencia emocional (Tabla 1). Se encontraron diferencias significativas en cuanto al género en todas las variables, excepto en comprensión de las emociones.

En los distintos factores de humor, las puntuaciones indican un mayor uso de éste en sus cuatro factores por parte de los chicos. Mientras, en la gestión de la emociones, en la investigación, los chicos prestaban una mayor atención y regulación de esas emociones que las chicas.

Tabla 1. Resultados obtenidos por género (en \%) en las escalas HSQ y TMMS-24

\begin{tabular}{llcccccc} 
& & \multicolumn{3}{c}{ Hombres } & Mujeres & $T$ & Signif. \\
\hline HSQ & Media & $d s$ & Media & $d s$ & & \\
& Humor afiliativo & 33.62 & 3.92 & 31.44 & 4.47 & 4.786 & .000 \\
& Humor automejora & 28.29 & 5.00 & 25.99 & 5.03 & 4.397 & .000 \\
& Humor agresivo & 21.01 & 3.58 & 18.56 & 3.87 & 6.139 & .000 \\
& Humor & 20.08 & 4.74 & 16.36 & 4.25 & 8.212 & .000 \\
& autodestructivo & & & & & & \\
\hline TMMS- & Atención & 31.27 & 4.78 & 28.79 & 5.06 & 4.750 & .000 \\
& & & & & & & \\
& Comprensión & 27.11 & 4.80 & 27.20 & 5.59 & .146 & .884 \\
& Regulación & 30.56 & 6.08 & 28.29 & 5.28 & 4.014 & .000 \\
\hline
\end{tabular}

En la tabla 2 se presentan las correlaciones parciales entre la escala del Humor (HSQ) y la escala de Inteligencia Emocional (TMMS-24). Ambos constructos se mostraron relacionados. La atención, estableció correlaciones con humor afiliativo, agresivo y autodestructivo, si bien con estos dos últimos la relación era inversa, indicando como una mayor atención de la emociones conlleva un menor uso del humor negativo. En cuanto a la 
comprensión, se relacionó también con el uso del humor afiliativo y de manera inversa con el uso agresivo y autodestructivo del mismo. Respecto a la regulación, mostró relación con los cuatro estilos del humor, observándose como la puntuación más alta se encontró entre el humor de automejora y la regulación de las emociones $\left(r=.666^{* *}\right)$, indicando que una mayor regulación de las emociones viene acompañada de un mayor uso del humor, tanto en sus estilos positivos (afiliativo y automejora) como negativos (agresivo $y$ autodestructivo).

Tabla 2. Correlaciones entre las escalas del humor e inteligencia emocional $(\mathrm{N}=1304)$

HSQ

Afiliación Automejora Agresividad Autodestructivo

\begin{tabular}{|c|c|c|c|c|c|}
\hline \multirow{3}{*}{$\sum_{i=}^{\stackrel{N}{N}}$} & Atención & $.120 * *$ & $-.119 * *$ & $-.108 * *$ & .020 \\
\hline & Comprensión & $.120 * *$ & .058 & $-.156 * *$ & $-.255^{* *}$ \\
\hline & Regulación & $.190 * *$ & $.666 * *$ & $.121 * *$ & $.128 * *$ \\
\hline
\end{tabular}

Por último, se realizó un análisis de clúster (Tabla 3) para intentar clasificar a los participantes en la investigación en función de las variables uso del humor e inteligencia emocional. De esta manera, se obtuvieron tres grupos: 1) formado por 417 adolescentes (31.98\% de la muestra), con niveles altos en el uso de los humores positivos (afiliación y automejora) y en las puntuaciones de inteligencia emocional; 2) con 516 alumnos (39.57\%), con niveles más bajos en todos los apartados, tanto en el uso del humor, como en inteligencia emocional; y 3) con 371 adolescentes (28.45\%) con mayor regulación de la emociones pero menor comprensión y atención a éstas y niveles más elevados en el humor de automejora y los humores negativos (agresivo y autodestructivo).

Tabla 3. Centro de conglomerados finales de las variables uso del humor e inteligencia emocional.

\begin{tabular}{llcccc}
\hline HSQ & & 1 & 2 & 3 & $x$ \\
& Afiliativo & $33.80^{\mathrm{a}}$ & $30.16^{\mathrm{b}}$ & 31.88 & 31.81 \\
& Automejora & $28.07^{\mathrm{a}}$ & $22.35^{\mathrm{b}}$ & $30.10^{\mathrm{a}}$ & 26.38 \\
& Agresivo & 18.60 & $17.67^{\mathrm{b}}$ & $21.25^{\mathrm{a}}$ & 18.98 \\
& Autodestructivo & $15.97^{\mathrm{b}}$ & $15.64^{\mathrm{b}}$ & $20.05^{\mathrm{a}}$ & 16.99 \\
\hline TMMS-24 & Atención & $32.15^{\mathrm{a}}$ & $28.27^{\mathrm{b}}$ & $27.23^{\mathrm{b}}$ & 29.21 \\
& Comprensión & $32.63^{\mathrm{a}}$ & $25.18^{\mathrm{b}}$ & $23.83^{\mathrm{b}}$ & 27.18 \\
& Regulación & $30.91^{\mathrm{a}}$ & $24.03^{\mathrm{b}}$ & $32.66^{\mathrm{a}}$ & 28.68 \\
\hline & $\mathrm{N}(\%)$ & $417(31.98 \%)$ & $516\left(39.57^{\circ}\right)$ & $371(28.45 \%)$ & \\
\hline
\end{tabular}

\footnotetext{
${ }^{a}$ Valores por encima de la media

${ }^{\mathrm{b}}$ Valores por debajo de la media
} 
Además, se intentó determinar la influencia que sobre las dimensiones del TMMS-24 tienen las variables demográficas y las dimensiones del humor. Para ello, se realizaron modelos de regresión múltiple jerárquicos que incluían como variables predictoras, en el paso 1, las variables demográficas género y edad $\mathrm{y}$, en el paso 2, las dimensiones del humor. $\mathrm{A}$ continuación, se muestran los resultados obtenidos.

En primer lugar, se analizaron las correlaciones entre las dimensiones del uso del humor y de la inteligencia emocional (Tabla 4). Se puede apreciar como no existe correlación entre la variable atención y los distintos factores del humor. Por otra parte, la comprensión sólo presenta correlación negativa con el humor contraproducente, y es la regulación quien correlaciona con los usos del humor positivo (afiliativo y de automejora).

Tabla 4. Correlaciones de las dimensiones Humor y TMMS24.

\begin{tabular}{|c|c|c|c|c|c|c|}
\hline Variable & 1 & 2 & 3 & 4 & 5 & 6 \\
\hline 1. Humor afiliativo & 1 & & & & & \\
\hline 2. Humor automejora & $.340 * *$ & 1 & & & & \\
\hline 3. Humor agresivo & .152 & $.180^{*}$ & 1 & & & \\
\hline $\begin{array}{ll}4 . & \text { Humor } \\
\text { contraproducente }\end{array}$ & .099 & $.202 *$ & $.341^{* *}$ & 1 & & \\
\hline 5. Atención & .121 & -.115 & .109 & .001 & 1 & \\
\hline 6. Comprensión & .111 & .066 & -.149 & $-.240 * *$ & $.258 * *$ & 1 \\
\hline 7. Regulación & $.184^{*}$ & $.664^{* *}$ & .123 & .128 & -.010 & $.268 * *$ \\
\hline
\end{tabular}

En las tablas 5 y 6 se muestra el resultado del modelo realizado sobre las dimensiones comprensión y regulación del TMMS-24 (el modelo para la dimensión atención no fue significativo). En ambas dimensiones, las variables demográficas no presentaron efecto significativo sobre ellas (paso 1). En comprensión (Tabla 5), con la adición en el modelo (paso 2) de las dimensiones del humor se produjo un aumento significativo de la varianza explicada del 9.5\%, siendo el total de la misma del 12.1\%. En este modelo, el humor contraproducente mostró un efecto indirecto y significativo $(B=.22, p=.005)$ con la comprensión por lo que la puntuación en comprensión se asocia a niveles bajos de dicho humor.

Por otra parte, en regulación (Tabla 6) se produjo un aumento significativo de la varianza explicada del $42.3 \%$, siendo el total de la misma del $44.5 \%$ con la adición en el modelo (paso 2) de las dimensiones del humor, siendo el humor de automejora el que mostró un efecto directo y significativo $(B=.53, p<.001)$ con la regulación, por lo que la puntuación en regulación se asocia a niveles altos de dicho humor. 
Tabla 5. Análisis de regresión múltiple sobre comprensión.

\begin{tabular}{|c|c|c|c|c|c|c|}
\hline \multirow[b]{2}{*}{ Predictor } & \multicolumn{3}{|c|}{ Paso 1} & \multicolumn{3}{|c|}{ Paso 2} \\
\hline & $B(E T)$ & $t$ & r parcial & $B(E T)$ & $t$ & $\begin{array}{c}\mathbf{r} \\
\text { parcial }\end{array}$ \\
\hline Género & $0.43(1.21)$ & 0.35 & 0.03 & $-0.47(1.27)$ & -0.37 & -0.03 \\
\hline Edad & $0.17(0.09)$ & 1.91 & 0.16 & $0.17(0.09)$ & 1.95 & 0.17 \\
\hline 1. Humor afiliativo & & & & $0.11(0.08)$ & 1.47 & 0.13 \\
\hline 2. Humor automejora & & & & $0.07(0.07)$ & 0.99 & 0.09 \\
\hline 3. Humor agresivo & & & & $-0.08(0.09)$ & -0.92 & -0.08 \\
\hline 4. Humor contraproducente & & & & $-0.22(0.08)$ & $-2.83^{* *}$ & -0.24 \\
\hline$R^{2}(\%)$ & \multicolumn{3}{|c|}{2.6} & \multicolumn{3}{|c|}{12.1} \\
\hline$\Delta \mathbf{R}^{2}(\%)$ & & & & \multicolumn{3}{|c|}{9.5} \\
\hline Modelo & \multicolumn{3}{|c|}{$F=1.82$} & \multicolumn{3}{|c|}{$\mathrm{F}=2.99 * *$} \\
\hline
\end{tabular}

Tabla 6. Análisis de regresión múltiple sobre regulación.

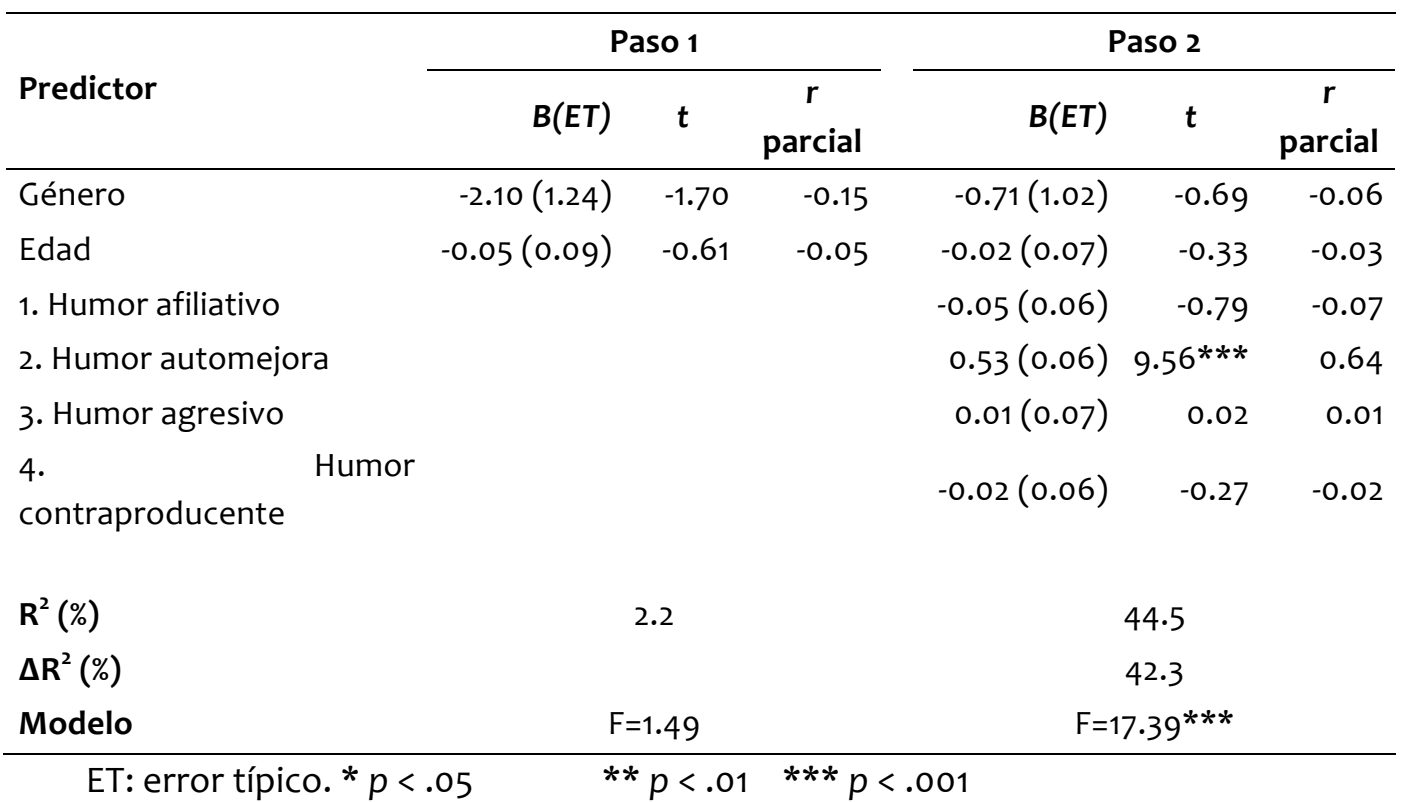

\section{Discusión y conclusiones}

Los datos de la investigación indican como los chicos hacen un uso más elevado de los distintos estilos del humor, tantos positivos como negativos, aun cuando no existen suficientes investigaciones sobre este tema como para poder extraer conclusiones firmes acerca de la relación del género con el uso del humor (Dick y Holtzman, 2013; Hay, 2000; Kotthoff, 2006). También se encontró un mejor manejo de las emociones en los chicos en cuanto a atención y a regulación, pero una menor comprensión en esas emociones. En este punto, sorprende que las chicas obtengan puntuaciones inferiores en inteligencia 
emocional y supone una diferencia con otros estudios que no encontraron diferencias en cuánto al uso las emociones en cuestión de género (Batool, Niazi y Ghayas, 2014), o que señalaban un mejor manejo de las emociones en el caso de las chicas (Extremera, Fernández-Berrocal y Salovey, 2006; Fortes-Vilaltella, Oriol, Filella, Del Arco, \& Soldevila, 2013). Esto quizás pueda deberse al uso en la presente investigación de una escala autoinformada, como es el TMMS-24, y debería indicar la necesidad del uso de un instrumento de medida más fiable en este sentido, como el MSCEIT (Mayer, Salovey \& Caruso, 2009). Otros autores, (Gartxia, Aritzeta, Bulluerka y Barberá, 2012), plantean que las diferencias por género encontradas en cuánto a inteligencia emocional, pueden ser debidas a la identidad de género y las puntuaciones más altas en las chicas, no ser un resultado de una cuestión de la naturaleza, sino de la educación, haciendo necesario trabajar estos aspectos en los estudiantes de Secundaria con programas y tareas de educación emocional en nuestras escuelas.

En cuanto a la relación existente entre los estilos del humor y la inteligencia emocional, los resultados indican cómo la atención de las emociones mantiene relación con el humor afiliativo. Esta atención y la comprensión, señalan una relación inversa con los estilos negativos del (agresivo y autodestructivo), es decir, cuanto mayor atención y comprensión mantiene la persona de sus emociones se produce un menor uso de esos estilos de humor negativos. La regulación de esas emociones correlacionó con los cuatro estilos de humor, tanto positivos como negativos, es decir, a mayor regulación, mayor manejo de los diferentes estilos de humor.

Los resultados de estas relaciones entre estilos del humor e inteligencia emocional, se pueden explicar si analizamos el uso que las personas hacen de los diferentes estilos del humor. Así, el humor afiliativo (o humor social) mide el uso benigno del humor para facilitar las relaciones interpersonales, el cual mostró relación con los tres factores de IE (atención, comprensión y regulación) en línea de otros datos encontrados en otras investigaciones anteriores (Erickson \& Feldstein, 2007; Kuiper \& McHale, 2009). El humor de automejora, que se usa como una estrategia de afrontamiento para regular las emociones negativas, estuvo relacionado con la regulación y atención de la emociones, indicando que un uso de este humor, implica una menor atención a las emociones y una mayor regulación de las mismas, en línea con otros estudios (Saroglou \& Scariot, 2002). El humor agresivo, que es utilizado para sobresalir a expensas de otros, correlacionó con una mayor regulación y una menor atención y comprensión de las emociones, cuestión ya apuntada por Kuiper \& McHale (2009). El humor autoagresivo, que consiste en hacer o decir cosas despectivas o ridiculizarse a sí mismo para hacer reír a otros, se encontró relacionado con una mayor regulación y una menor comprensión de las emociones en el mismo sentido del estudio de Aillaud \& Piolat (2013). Por último, indicar que se observó que los dos factores más relacionados serían la regulación de las emociones y el humor de automejora, que indicaría que las personas que mejor regulan las emociones, utilizan este humor como una herramienta útil para relacionarse con otras personas.

Por otra parte, al realizarse el análisis de clúster, se obtuvieron tres grupos: el primero, compuesto por adolescentes con niveles altos en el uso de los humores positivos (afiliación y automejora) y en las puntuaciones de inteligencia emocional; el segundo, formado por 
niveles más bajos en todos los apartados, tanto en el uso del humor, como en inteligencia emocional; y, por último, un tercero con una mayor regulación de la emociones aunque con una menor comprensión y atención a éstas y niveles más altos en el humor de automejora y los humores negativos (agresivo y autodestructivo). Estos resultados, indican que un $39.57 \%$ de los adolescentes muestran bajos niveles de inteligencia emocional y del uso del humor en sus relaciones; un segundo grupo (31.98\%), hacen un buen uso del humor, con el empleo de los estilos de humor positivo y un bajo uso de los negativos, así como una alta inteligencia emocional; y un tercer grupo (28.45\%) regulan bien sus emociones, pero esta regulación la emplean para hacer un menor uso del humor y mostrar una menor atención y comprensión de sus emociones. Se observó que un 68.02\% de los adolescentes muestran bajos niveles de inteligencia emocional y del uso del humor, lo que señalaría la necesidad de implementar programas de intervención para entrenar e incentivar a los adolescentes en un buen uso del humor, así como programas de educación emocional en el aula, que les puedan proporcionar soporte emocional y servir en la gestión de conflictos dentro y fuera del aula.

Respecto a la influencia que tienen las variables demográficas y las dimensiones del humor sobre las dimensiones del TMMS-24, los resultados mostraron correlaciones entre el uso del humor y la inteligencia emocional de los sujetos de la muestra. El modelo para la dimensión atención no fue significativo. Tampoco género o edad presentaron efectos significativos. En comprensión, el humor contraproducente presentó un efecto indirecto y significativo, lo que indicaría que una puntuación alta en comprensión, se asociará a niveles bajos en el uso de dicho humor. En cuanto a la regulación, el humor de automejora mostró un efecto directo y significativo, lo que indicaría que niveles altos en regulación se asociarán a un uso de este humor.

Los resultados de la presente investigación deben ser interpretados teniendo en cuentas las limitaciones de este estudio; si bien la muestra es relevante a nivel estadístico, debería ampliarse a otros estratos de la población, incluidas poblaciones clínicas, dónde podría analizarse la asociación entre estos dos constructos: uso del humor e inteligencia emocional. También serían deseables estudios longitudinales que permitiesen valorar la evolución del uso del humor, así como su vinculación con la inteligencia emocional, a lo largo de un período más largo de tiempo.

De cualquier forma, los resultados de este trabajo, permiten concluir que el constructo de uso del humor medido con el HSQ establece adecuadas correlaciones con la inteligencia emocional percibida (TMMS-24), proporcionando los datos de la investigación evidencia de la relación entre estas variables objetos de estudio.

Las aportaciones del presente estudio residen en la necesidad de la implementación de programas para trabajar ambos constructos objeto de estudio de nuestro trabajo en el aula reconociendo, de este modo, el enorme potencial educativo que puede suponer para los discentes, profesores y comunidad educativa en conjunto encaminándonos a la educación integral del alumnado. 


\section{Referencias}

Aillaud, M., \& Piolat, A. (2013). Compréhension et appreciation de l'humour: aproche cognitivoémotionnelle. Psychologie française, 58, 255-275. doi:10.1016/j.psfr.2013.04.001

Airenti, G. (2016). Playing with expectations: A contextual view of humor development. Frontiers in Psychology, 7, 1392. doi:10.3389/fpsyg.2016.01392

Ato, M., y Vallejo. G. (2015). Diseños de investigación en Psicología. Madrid: Pirámide.

Batool, M., Niazi, S., \& Ghayas, S. (2014). Emotional intelligence as a predictor of sense of humor and hope among adults. Journal of the Indian Academy of Applied Psychology, 40, 270-278.

Berrios-Martos, M.P., Pulido-Martos, M., Augusto-Landa, J.M., y López-Zafra, E. (2012). La inteligencia emocional y el uso del humor como variables predictoras del bienestar subjetivo. Behavioral Psychology, 20, 211-217.

Caballero, M.F., Alcaraz, V., Alonso, J.I., \& Yuste, J.L. (2016). Intensidad emocional en la clase de educación física en función de la victoria: juegos de cooperación-oposición. Revista Electrónica Interuniversitaria de Formación del Profesorado, 19(3), 123-133. doi: 10.6018/reifop.19.3.267291

Cann, A., \& Etzel, K. (2008). Remembering and anticipating stressors: Positive personality mediates the relationship with sense of humor. Humor: International Journal of Humor Research, 2, 157178.

Capps, D. (2006). The psychological benefits of humor. Pastoral Psychology, 54, 393-411.

Carbelo, B. (2011). Un nuevo enfoque en valores: el humor y la alegría en el ámbito de la educación. Revista Padres y Maestros/Journal of Parents and Teachers, 337, 17-20.

Carretero-Dios, H., Benítez, I., Delgado-Rico, E., Ruch, W., \& López-Benítez, R. (2014). Temperamental basis of sense of humor: The Spanish long form of the trait version of the State-TraitCheerfulness-Inventory. Personality and Individual Differences, 68, 77-82. doi:10.1016/j.paid.2014.03.045

Cassaretto, M., y Martínez, P. (2009). Validación de la Escala del Uso del humor en estudiantes universitarios. Revista de Psicología, 27, 287-309.

Chen, G.H., \& Martin, R. (2007). A comparision of humor styles, coping humor, and mental Health between Chinese and Canadian university students. Humor, 20, 215-234.

Di Fabio, A., \& Kenny, M.E. (2016). Promoting well-being: The contribution of emotional intelligence. Frontiers in Psychology, 7(AUG), 01182. doi:10.3389/fpsyg.2016.01182

Di Fabio, A., \& Saklofske, D.H. (2014). Comparing ability and self-report trait emotional intelligence, fluid intelligence, and Personality traits in career decision. Personality and Individual Differences, 64, 174-178. doi:10.1016/j.paid.2005.02.004

Dyck, K., \& Holtzman, S. (2013). Understanding humor styles and well-being: the importance of social relationships and gender. Personality and Individual Differences, 55, 53-58. doi:10.1016/j.paid.2013.01.023

Edgar, C., McRorie, M., \& Sneddon, I. (2012). Emotional intelligence, personality and the decoding of non-verbal expressions of emotions. Personality and Individual Differences, 52, 295-300. doi:10.1016/j.paid.2011.10.024 
Erickson, S., \& Feldstein, S. (2007). Adolescent humor and its relationship to coping, defense strategies, psychological distress, and well-being. Child Psychiatry and Human Development, 37, 255-271.

Extremera, N., \& Rey, L. (2016). Ability emotional intelligence and life satisfaction: Positive and negative affects as mediators. Personality and Individual Differences, 102, 98-101. doi:10.1016/j.paid.2016.06.051

Extremera, N., Fernández-Berrocal, P., \& Ramos, N. (2004). Validity and reliability of the Spanish modified version on the Trait Meta-Mood Scale. Psychological Reports, 94, 751-755.

Extremera, N., Fernández-Berrocal, P., y Salovey, P. (2006). Spanish version of the Mayer-SaloveyCaruso Emotional Intelligence Test (MSCEIT). Version 2.0: reliabilities, age and gender differences. Psicothema, 18, 42-48.

Ford, T.E., Lappi, S.K., \& Holden, C.J. (2016b). Personality, humor styles and happiness: Happy people have positive humor styles. Europe's Journal of Psychology, 12, 320-337. doi:10.5964/ejopv12i31160

Fortes-Vilaltella, M., Oriol, X., Filella, G., Del Arco, I., \& Soldevila, A. (2013). Inteligencia emocional y personalidad en las diferentes áreas de conocimiento de los estudiantes universitarios. Revista Electrónica Interuniversitaria de Formación del Profesorado, 16(1), 109-120. doi: 10.6018/reifop.16.1.179471

Fox, C.L., Hunter, S.C., \& Jones, S.E. (2016). Children's humor types and psychosocial adjustment. Personality and Individual Differences, 89, 86-91.

Fox, L., Hunter, C., \& Jones, S.E. (2016a). Longitudinal associations between humor styles and psychosocial adjustment in adolescence. Europe's Journal of Psychology, 12, 377-389. doi:10.5964/ejop.v12i3.1065

Freudenthaler, H.H., \& Neubauer, A.C. (2005). Emotional intelligence: The convergent and discriminant validities of intra- and interpersonal emotional abilities. Personality and Individual Differences, 39, 569-579.

Garay, B., Mendiguren, H., Álvarez, A. \& Vizcarra, M.T. (2017). Los significados otorgados a las relaciones adolescentes desde la comunidad escolar. Revista Electrónica Interuniversitaria de Formación del Profesorado, 20(3), 179-194. doi: 10.6018/reifop.20.3.270711

García-Larrauri, B., Manzano, M. E., Muñoz, M. I., Hernández, M. y Cuetos, A. (2010). Claves para aprender en un ambiente positivo y divertido. Todo rueda mejor si se engrasa con humor. Madrid: Pirámide.

Gartxia, L., Aritzeta, A., Bulluerka, N., y Barberá, E. (2012). Inteligencia emocional y género: más allá de las diferencias sexuales. Anales de Psicología, 28, 567-575. doi:10.6018/analesps.28.2.124111

Gignac, G.E., Karatamoglou, A., Wee, S., \& Palacios, G. (2014). Emotional intelligence as a unique predictor of individual differences in humour styles and humour appreciation. Personality and Individual Differences, 56, 34-39. doi:10.1016/j.paid.2013.08.020

Hay, J. (2000). Emotions of humor in the conversations of men and women. Journal of Pragmatics, 32, 709-742.

Heintz, S. (2017). Putting a spotlight on daily humor behaviors: Dimensionality and relationships with personality, subjective well-being, and humor styles. Personality and Individual Differences, 104, 407-412. 
Kotthoff, H. (2006). Gender and humor: the state of the art. Journal of Pragmatics, 38, 4-25. doi:10.1016/j.pragma.2005.06.003

Kozbetz, A., \& Nishioka, K. (2010). Humor comprehension, humor production, and insight: An exploratory study. Humor: International Journal of Humor Research, 23, 375-401.

Kuiper, N.A., \& McHale, N. (2009). Humor styles as mediators between self-evaluative standards and psychological well-being. Journal of Psychology, 143, 359-376. doi:10.3200/JRLP.143.4.359-376

Kuiper, N.A., Grimshaw, M., Leite, C., \& Kirsh, G. (2004). Humor is not always the best medicine: specific components of sense of humor and psychological well-being. Humor: International Journal of Humor Research, 17, 135-168. doi:10.1515/humr.2004.002

Lefcourt, H.M. (2001). Humor: the psychology of living buoyantly. New York: Kluwer Academic.

Lopes, P.N., Brackett, M.A., Nezlek, J.B., Schutz, A., Sellin, I., \& Salovey, P. (2004). Emotional intelligence and social interaction. Personality and Social Psychology Bulletin, 30, 1018-1034.

López-Pérez, B., Wilson, E.L., Dellaria, G., \& Gummerum, M. (2016). Developmental differences in children's interpersonal emotion regulation. Motivation and Emotion, 40, 767-780. doi: $10.1007 / \mathrm{s} 11031-016-9569-3$

Maiolino, N.B., \& Kuiper, N.A. (2014). Integrating humor and positive psychology approaches to psychological well-being. Europe's Journal of Psychology, 10, 557-570. doi:10.5964/ejop.v10i3.753

Martin, R.A. (2001). Humor, laughter, and physical health: methodological issues and research findings. Psychological Bulletin, 127, 504-519.

Martin, R.A. (2008). Psicología del humor. Un enfoque integrador. Madrid: Orión.

Martin, R.A., Kuiper, N.A., Olinger, L.J., \& Dance, K.A. (1993). Humor, coping with stress, self-concept, and psychological well-being. Humor: International Journal of Humor Research, 6, 89-104.

Martin, R.A., Puhlik-Doris, P., Larsen, G., Gray, J., \& Weir, K. (2003). Individual differences in uses of humor and their relation to psychological well-being: Development of the humor styles questionnaire. Journal of Research in Personality, 37, 48-75.

Mayer, J.D., Salovey, P., \& Caruso, D.R. (2009). MSCEIT. Test de Inteligencia emocional de MayerSalovey-Caruso. Madrid: TEA Ediciones S.A.

Moran, J.M., Rain, M., Page-Gould, E., \& Mar, R.A. (2014). Do I amuse you? Asymmetric predictors for humor appreciation and humor production. Journal of Research in Personality, 49, 8-13. doi:10.1016/j.jrp.2013.12.002

Nezlek, J.B., \& Derks, P. (2001). Use of humor as a coping mechanism, psychological adjustment, and social interaction. Humor, 14, 395-413.

Panish, J. (2002). Life satisfaction in the elderly: the role of sexuality, sense of humor, and health. Dissertation Abstracts International, 63 (5-B).

Salavera, C., \& Usán, P. (2017). Repercusión de las estrategias de afrontamiento de estrés en la felicidad de los alumnos de Secundaria. Revista Electrónica Interuniversitaria de Formación del Profesorado, 20(3), 65-77. doi: 10.6018/reifop.20.3.282601

Saroglou, V., \& Scariot, C. (2002). Humor Style Questionnaire: Personality and educational correlates in Belgian high school and college students. European Journal of Personality, 16, 43-54. 
Sirigatti, S., Penzo, I., Giannetti, E., Casale, S., \& Stefanile, C. (2016). Relationships between humorism profiles and Psychological well-being. Personality and Individual Differences, 90, 219-224. doi:10.1016/j.paid.2015.11.011

Stough, C., Saklofske, D., \& Parker, J. (2009). Assessing Emotional Intelligence. New York: Springer.

Vrabel, J.K., Zeigler-Hill, V., \& Shango, R.G. (2017). Spiteful and humor styles. Personality and Individual Differences, 105, 238-243.

Weinstein, N., Hodgins, H.S., \& Ostvik-White, E. (2011). Humor as aggression: Effects of motivation on hostility expressed in humor appreciation. Journal of Personality and Social Psychology, 100, 1043-1055. doi:10.1037/a0022495

Yip, J.A., \& Martin, R.A. (2006) Sense of humor, emotional intelligence, and social competence. Journal of Research in Personality, 40, 1202-1220. doi:10.1016/j.jrp.2005.08.005

Ziv, A., \& Gadish, O. (1989). Humor and marital satisfaction. Journal of Social Psychology, 129, 759-768. 